\title{
TRANSFUSION REACTION AMONG THE BLOOD RECIPIENT - A STUDY OF 120 CASES
}

\author{
Chowdhury $\mathrm{FS}^{1}$, Biswas $\mathrm{J}^{2}$, Siddiqui MAE ${ }^{3}$, Hoque $\mathrm{MM}^{4}$, Adnan $\mathrm{SK}^{5}$
}

\begin{abstract}
:
Context: Blood transfusion is a life saving procedure. Transfusion of one unit of blood, plasma or platelet if employed safely, well in time with proper intensive care can save many valuable lives. But due to transfusion a number of untoward reactions develop that are sometimes more serious and life threatening So the aim of this study was to find out the most frequent and life threatening reactions that develop during transfusion.

Methods: In between January'2006 to December'2007 a total 120 subjects, mean aged 25.9 \pm 13.9 years were selected as study population in BSMMU. Those who were receiving transfusion for congenital hemolytic anemia, aplastic anemia, refractory anaemia, undiagnosed anaemia, anaemia of chronic disease, hematological malignancy, post chemotherapy, clotting factor deficiency, pre and post operatively as well as during operation. The transfusion unit was single unit to 100 unit of fresh whole blood or its component- red cell concentrate, fresh frozen plasma, platelet concentrate, cryoprecipitate.

Results: There were 8 (6.66\%) patients showed reactions of different types. Among them febrile reaction was 5 (62.5\%), allergic reaction was 2 (25\%) and 1 (12.5\%) developed pulmonary congestion (Transfusion Related Acute Lung Injury), characterized by cough, shortness of breath and increase rate of respiration. No fatal reaction observed during the study period.
\end{abstract}

Key words: Transfusion reaction, Febrile reaction, Allergic reaction.

J Dhaka Med Coll. 2008; 17(2) : 67-71.

\section{Introduction:}

Blood transfusion is the term used to describe the therapeutic use of all blood products. When used correctly, it has many clinical benefits and can be life saving but at the same time it also carries considerable risk.

A transfusion reaction is any unfavorable transfusion related event occurring in a patient during or after transfusion of blood and blood component. It is estimated that $2-5 \%$ of transfusion are associate with adverse events, most of which are minor and are dealt with at bed side.

Transfusion reactions are immunogenic and nonimmunogenic, acute or delayed. Acute reactions occur within 24 hours. Acute reactions are cell and plasma related and are immediate hemolytic transfusion reaction
(IHTR), febrile nonhemolytic reaction (FNHTR), urticarial, anaphylactic, transfusion related lung injury (TRALI), transfusion related sepsis, circulatory overload, nonhemolytic hemolysis, air embolism, hypothermia.

Delayed reactions usually occur after 24 hours. Delayed reactions are due to secondary anamnestic response and these are delayed hemolytic transfusion reaction (DHTR), alloimmunization against $\mathrm{RBC}$ and HLA antigen graft versus host disease, post transfusion purpura, immunodilution and iron overload.

Febrile non-hemolytic transfusion reaction is the most commonly encountered type of transfusion reaction. It may be defined as any $1 \mathrm{p} \mathrm{c}$ or greater temperature increase above the patient's baseline temperature during or

1. Assistant Registrar, Department of Transfusion Medicine, DMCH, Dhaka

2. Professor and Chairman, Department of Transfusion Medicine, BSMMU, Dhaka

3. Consultant, Cardiology, NITOR, Dhaka

4. Assistant Professor, Department of Transfusion Medicine, DMCH, Dhaka

5. Medical Officer, Department of Transfusion Medicine, DMCH, Dhaka

Correspondence: Dr. Fahmida Sharmin Chowdhury 
within 24 hours after transfusion. It is caused by antileukocyte antibodies present in the patient's serum. Alloimmunizaton by prior blood transfusion, tissue transplantation, or pregnancy is the causative stimulus for antibody formation. These antibodies are predominantly HLA antibodies. Febrile reaction may follow activation of the complement system, producing $\mathrm{C}_{5 \mathrm{a}}$ which causes production and release of pyrogen IL-1 from patient's macrophages and monocytes. IL-1 may initiate synthesis of $\mathrm{PGE}_{2}$ in the hypothalamic cells resulting in an additional pyrogenic effect. It is characterized by fever with or without chills. Severe reactions may include hypotension, cyanosis, tachycardia, tachypnea, dyspnea, cough, limited fibrinolysis and transient leucopenia.

Allergic reaction occurs in transfusion of whole blood, plasma or cell. Most allergic reactions are produced due to presence of antibody to donor's protein in recipient's blood. Donor plasma has foreign protein (allergen) with which IgE or IgG or both react. These antigen antibody complex attaches to the mast cells and cause degranulation of mast cell and release of histamine, serotonin and leukotrienes. Majority of allergic reactions are mild and characterized by hives, rash often with itching. Urticaria is the commonest allergic reaction to blood but angioneurotic edema, asthma and even anaphylactic shock have been observed. Some severe form of anaphylactic shock are due to interaction between transfused IgA and class specific anti IgA in recipients plasma but in many cases the cause of reaction is unknown. Anaphylactic reactions are sudden in onset with pronounced symptoms that may include coughing, dyspnea, nausea, vomiting, brochospasm, flushing of skin, chest pain, hypotension loss of consciousness and death.

There is pulmonary complication known as transfusion related acute lung injury (TRALI). The cause is not well understood. It caused by interaction between leukocyte and antileukocyte antibody resulting complement activation and pulmonary vascular permeability. It is characterized by fever, chills, cough, cyanosis, hypotension and bilateral pulmonary edema and increasing respiratory distress shortly after transfusion of blood components volume that usually donot produce circulatory overload. Chest X-ray shows bilateral pulmonary infiltrate without cardiac enlargement. Clinical signs and symptoms may be mild resolving after a few days or severe resulting in rapidly progressive pulmonary failure.

\section{Methodology:}

In a simple observational study, total 200 patients were screened and followed up before, during and after transfusion in day care centre in transfusion medicine department of Bangabandhu Sheikh Mujib Medical University, Dhaka, during the period of January 2006 to December 2007. Patient under 5 years and over 65 years, seriously ill and debilitated patients with anemia or clotting factor deficiency were excluded and patients aged between 5 to 65 years, received blood or blood component, with single or multiple transfusions, were included, 120 patients were selected for this study. All patients selected were thoroughly evaluated by history and clinical examination. Baseline characteristics including age, sex, were recorded, relevant clinical information like, pulse, BP, temperature, anemia, jaundice, cyanosis, edema, dehydration, respiration rate, transfusion related other relevant data were recorded in a preformed data collection sheet. Close supervision with regular monitoring was done during and 2-3 hours after completion of transfusion.

\section{Results:}

Results are shown in tables and diagram.

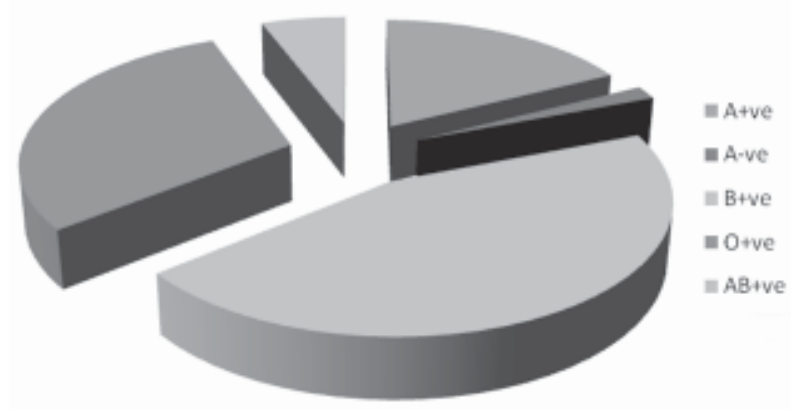

Fig.-I: Distribution ABO blood group 


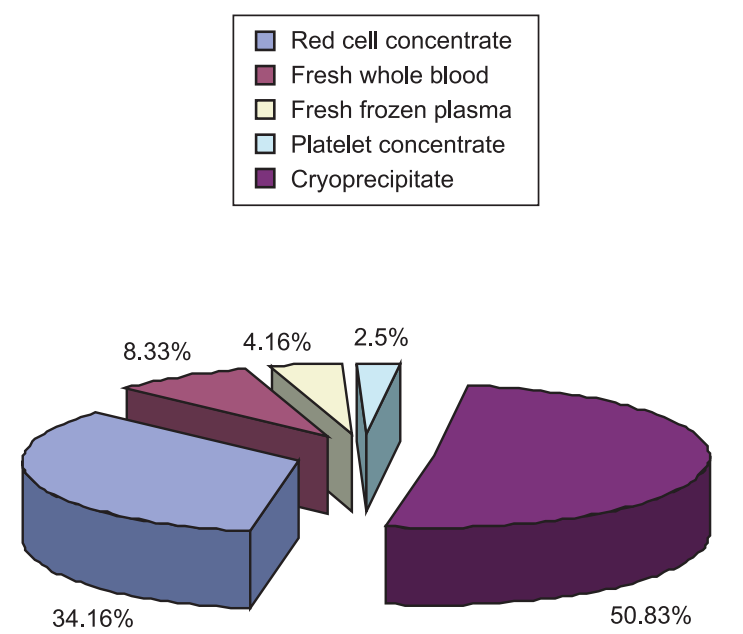

Fig.-2: Distribution of blood component

Table I

Ditribution of disease

\begin{tabular}{lcc}
\hline Name of Diseases & Frequency & Percentage \\
\hline Thalassaemia & 39 & 32.5 \\
Anaemia (aplastic,refactory, & 21 & 17.5 \\
undiagnosed, anaemia of & & \\
chronic diseaes & & \\
Haematological malignancy & 17 & 14.16 \\
Clotting factor defficiency & 10 & 8.33 \\
Post Chemoherapy & 14 & 11.66 \\
Gynaecological & 12 & 10 \\
Cardiac surgery & 7 & 5.83 \\
\hline Total & 120 & 100 \\
\hline
\end{tabular}

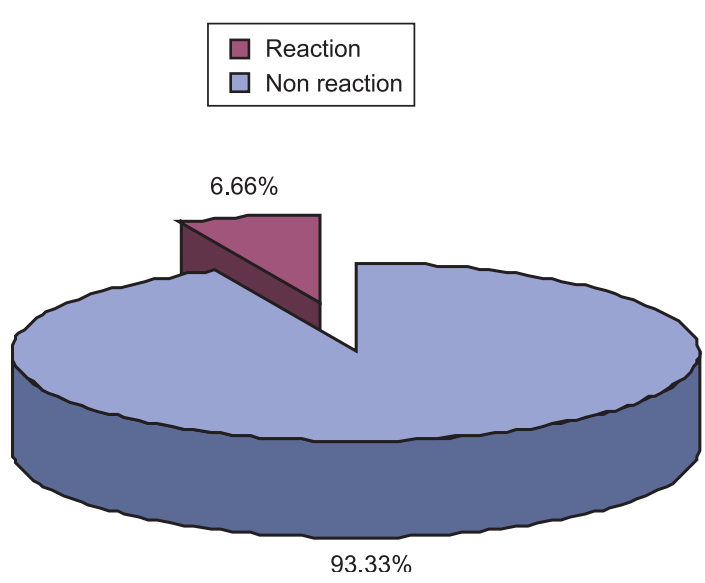

Fig.-3: Distribution of Transfusion reactions

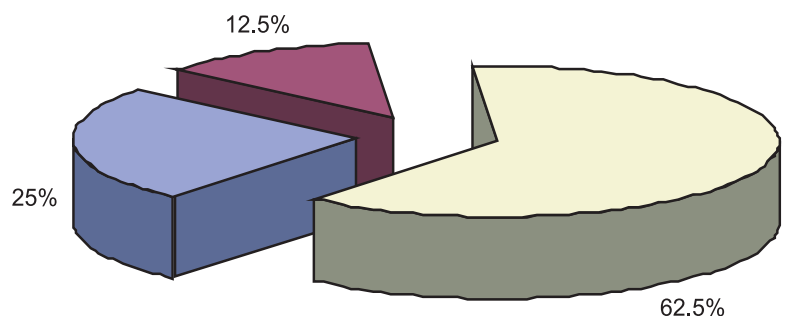

Fig.-4:Distibution of types of transfusion reactions.

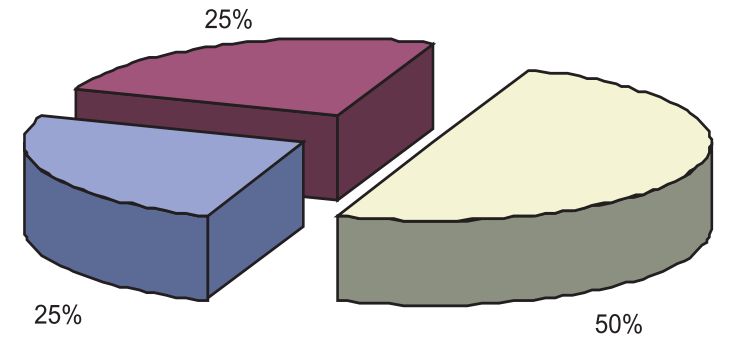

Fig.-5: Distibution of transfusion reaction by blood component

Table II

Distribution of transfusion reaction by unit of transfusion

\begin{tabular}{lc}
\hline Unit of transfusion & $\begin{array}{c}\text { Frequency of } \\
\text { transfusion reaction }\end{array}$ \\
\hline 1 to 20 & 01 \\
20 to 40 & 01 \\
40 to 60 & 01 \\
60 to 80 & 02 \\
80 to 100 & 03 \\
\hline Total & 08 \\
\hline
\end{tabular}




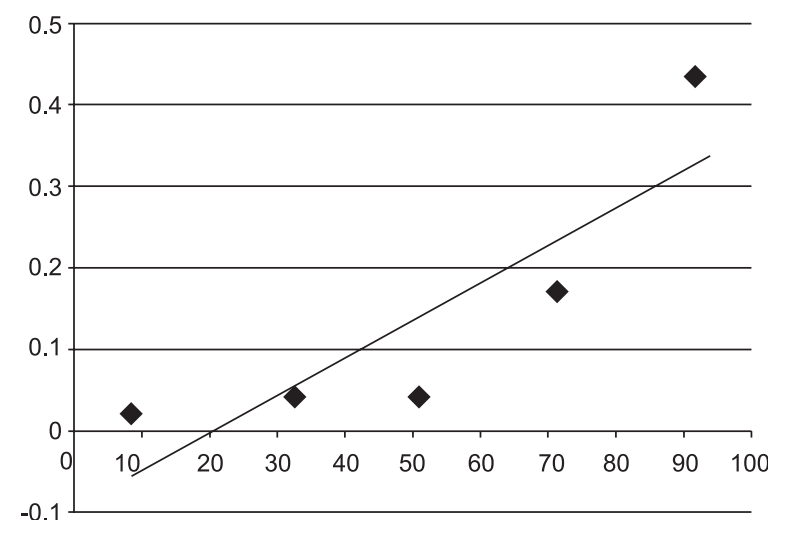

Fig.-6: Correlation between frequency of transfusion and probability of reaction.

There was a strong positve relationship between transfusion reaction and unit of transfusion with Pearson's correlation coefficient ( $r=0.86)$ (Fig.-6).

\section{Discussion:}

This simple observational study was carried out among 120 patient receiving transfusion of different component of blood in different types of diseases. This study was done to assess and explore the number and type of adverse effects of blood transfusion.

The patients were selected irrespective of age, sex and diagnosis. The study was based on history taking, physical examination before and after transfusion. A total number of 120 patients between 5 years to 65 years were studied. The mean age of patients was 25.91 year and SD was 13.89. Out of 120 attending patients $81(87.50 \%)$ were male and 39 (32.50\%) were female.

In this study most of the patients $54(45 \%)$ were group B, 36 (30\%) were group O, 23 (19.16\%) were group A and 7 (5.83\%) were Group AB. In $\mathrm{Rh} \mathrm{D}$ typing only $2(1.6 \%)$ were $\mathrm{Rh} \mathrm{D}$ negative and rest 118 (98.41\%) were Rh D positive.

This study revealed that $39(32.5 \%)$ were cases of thalassaemia, 21 (17.5\%) were anaemia, 17 $(14.16 \%)$ were haematological malignancy, 14 (11.66\%) were post chemotherapy, 12 (10\%) were gynaecological , 10 (8.33\%) were clotting factor deficiency, $7(5.83 \%)$ were cardiac surgery.
Out of 120 patients receiving blood and blood components, red cell concentrate was highest $61(50.83 \%)$ unit and cryoprecipitate was lowest $3(2.5 \%)$ unit. Fresh whole blood transfusion having second $41(34.16 \%)$ unit. Fresh frozen plasma was $10(8.33 \%)$ unit and platelet concentrates $5(4.16 \%)$ unit.

In this study the mostly used component was red cell concentrate $61(\%)$, the highest transfusion was in thalassaemia $73.95 \%$ which correlate with Shil's study ${ }^{1}$.

This study revealed that transfusion reaction occurred in $8(6.66 \%)$ cases out of 120 cases. In one study done by Rahman $(1977)^{2}$ total transfusion reactions were $10 \%{ }^{1}$. In another study done at BSMMU reactions were $8 \%$. The rate of transfusion reaction gradually decreasing, it may be due to improvement of quality.

Among the 8 (6.66\%) transfusion reaction, 5 $(62.5 \%)$ were febrile reaction. Fever usually come at the end of transfusion with chill and not exceeds $101^{\circ} \mathrm{F}$. It was managed by simple paracetamol. Shil et al. (2005) ${ }^{1}$ at BSMMU observed that febrile reaction were commonest $89.51 \%$, prevented and managed by simple paracetamol and steroid. Febrile reaction was best prevented by paracetamol or disprin orally half an hour before transfusion. Use of steroid before transfusion may musk mismatch transfusion. Units of blood causing febrile reaction can be transfused successfully. Use of filter is out of reach of most patients in Bangladesh. Washed red cell only required in case of severe hypotensive reaction.

In this study allergic reaction observed 2 (25\%). Reactions were mild and characterized by hives and some skin manifestation. No anaphylactic reaction or severe reaction occurred. Allergic reaction treated by simple chlorpheneramine.

Total 5 febrile reactions occurred out of 8 transfusion reaction. Their pre-transfusion temperature was $98.2 \pm 0.57$, pulse was $76.8 \pm$ 3.34 , systolic blood pressure was $112 \pm 8.36$, and diastolic blood pressure was $73 \pm 8.36$ and post transfusion temperature was $100.2 \pm 0.83$, pulse was $103.6 \pm 5.72$, systolic blood pressure was $119 \pm 7.41$ and diastolic blood pressure was $80 \pm$ 
6.12. Total change of temperature ( $\mathrm{p}-<0.001)$, pulse ( $\mathrm{p}-<0.001)$, systolic blood pressure ( $\mathrm{p}$ $<0.05$ ), and diastolic blood pressure ( $\mathrm{p}-<0.02)$ were statistically significant.

This study revealed that among total 8 transfusion reactions 1 (mean 0.02 ) reactions occurred at between 1 to 20 unit (mean $8.46 \pm 6.8$ ), 1 (mean 0.04 ) reaction occurred between 20 to 40 units (mean 32.92 \pm 5.7 ) and 1 (mean 0.04)reaction occurred between 40 to 60 units (mean 50.96 \pm 6.2 ), 2 (mean 0.17 ) reaction occurred between 60 to 80 units (mean $71.17 \pm 6.8$ ), 3(mean 0.43) reaction occurred between 80 to 100 units (mean 92 \pm 5.1 ). There was a strongly positve relationship between transfusion reaction and unit on transfusion (Pearson's correlation coefficient, $r=0.86$ ). That means increasing the number of transfusion, increasing the chance of reaction. Tan $\&$ Lee $(1993)^{3}$ found that febrile reactions were relatively common with repeated transfusion and tendency to develop reaction increase with the number transfusion increases leukocyte free red cell should be the treatment of choice for multiple transfused pateint as leukocyte are the important cause of transfusion reaction.

\section{Conclusion:}

During study period it was observed that blood transfusion reactions are related with frequency and unit of transfusion. Multi transfusion and transfusion of unwashed red cell concentrate contaminated with leukocyte and plasma protein were mainly responsible for febrile and allergic reaction. Washed red cell concentrate showed no such reaction during study period.

Maintaining a proper transfusion schedule and use of leukocyte depleted component and keeping transfusion minimum and only using when absolutely indicated may make blood transfusion safer and should be in clinical practice.

In addition to reduce transfusion reaction blood grouping, cross-matching and screening should be done properly and aseptic precaution should be taken during blood collection, storage and transfusion and maintenance of storage temperature and environment.

\section{Acknowledgement:}

Special thanks to all teachers, colleagues, fellow students and medical officers of Transfusion Medicine, BSMMU for their kind and sincere co-operation in this study.

\section{References:}

1. Shil N, Biswas J, Islam A, Khatun A. Day care transfusion therapy- one-year experiences in a day care transfusion unit of transfusion medicine department in a tertiary care hospital. Bangladesh J Med. 2005; 16: 83-6.

2. Rahman M. Transfusion reaction. Bangladesh Med J. 1977; 5: 81-5.

3. Tan KK, Lee WS, Liaw LCT. A prospective study on the use of leukocyte filters in reducing blood transfusion reactions in multitransfused thalassaemic children. Singapore Med J. 1993; 39: 109-11. 